\title{
ON SUBSPACES OF THE SPACE $(m)$
}

\section{J. COPPING}

In this note, $m, c$, and $c_{0}$ denote the Banach spaces of bounded, convergent, and null sequences, with the norm

$$
\|x\|=\sup _{n}\left|s_{n}\right| \quad\left(x=\left\{s_{n}\right\} \in m\right) .
$$

THEOREM. If $A$ is a given matrix summability method, $X_{0}$ the subspace of $m$ consisting of all bounded sequences summed to zero by $A$, and $c_{0}$ a proper subspace of $X_{0}$, then there exists no bounded projection of $X_{0}$ onto $c_{0}$.

Corollary. If $X$ is the space of atl bounded sequences summed by $A$, and $c$ a proper subspace of $X$, then there exists no bounded projection of $X$ onto $c$.

The corollary was conjectured by Wilansky $[6$, p. 250]; relevant known results are that $X_{0}$ and $X$ are nonseparable $([2,3.6 .2]$ and $[1, \mathrm{pp} .97-99]$ ), that there exists a bounded projection onto $c$ (or $c_{0}$ ) of any separable subspace of $m$ containing $c$ (or $c_{0}$ ) ([3, Theorem 2.2] and [5, Theorem 5]), and that there exists no bounded projection of $m$ onto $c$ or $c_{0}([4$, p. 539] and [5, p. 945]).

To prove the theorem, observe that by $[2,3.6]$, there exists a closed subspace $Y_{0}$ of $X_{0}$, a bounded linear operator $T$ from $m$ onto $Y_{0}$, and a strictly increasing sequence $\left\{m_{p}\right\}$ of positive integers, such that for $p=1,2, \cdots$,

$$
t_{m_{p}}=u_{p} \quad \text { for all }\left\{u_{n}\right\} \in m \text {, where } T\left(\left\{u_{n}\right\}\right)=\left\{t_{n}\right\} .
$$

The construction given in $[2,3.6]$ for this operator $T$ ensures also that $T\left(c_{0}\right) \subseteq c_{0}$.

Now suppose if possible that there exists a bounded projection $P$ of $X_{0}$ onto $c_{0}$. Let $Q_{1}: Y_{0} \rightarrow c_{0}$ be the restriction of $P$ to $Y_{0}$, and define the bounded linear operator $T_{1}=Q_{1} T: m \rightarrow c_{0}$. Since $T\left(c_{0}\right) \subseteq c_{0} \cap Y_{0}$,

$$
T_{1}\left(\left\{u_{n}\right\}\right)=T\left(\left\{u_{n}\right\}\right) \quad \text { when }\left\{u_{n}\right\} \in c_{0} .
$$

Define a bounded linear operator $R: c_{0} \rightarrow c_{0}$ by

(3) $v_{p}=s_{m_{p}} \quad$ for all $\left\{s_{n}\right\} \in c_{0}$, where $R\left(\left\{s_{n}\right\}\right)=\left\{v_{n}\right\}$,

for $p=1,2, \cdots$. Let $Q_{2}$ be the restriction of $R$ to the range of $T_{1}$,

Received by the editors August 13, 1963. 
and define $T_{2}=Q_{2} T_{1}: m \rightarrow c_{0}$. By (1), (2), and (3), if $\left\{u_{n}\right\} \in c_{0}$, and $T_{2}\left(\left\{u_{n}\right\}\right)=\left\{v_{n}\right\}$, then $u_{p}=v_{p}$ for $p=1,2, \cdots ;$ thus $T_{2}$ is a bounded projection of $m$ onto $c_{0}$. This contradicts the result of Sobczyk [5, p. 945], and the theorem is proved.

For the corollary, let $\left(a_{n, k}\right)$ be the matrix of the method $A$, and define a method $B$ by the matrix $\left(b_{n, k}\right)$, where

$$
b_{n, k}=a_{n, k}-\lim _{n \rightarrow \infty} a_{n, k}
$$

let

$$
\lambda(B)=\lim _{n \rightarrow \infty} \sum_{k=1}^{\infty} b_{n, k},
$$

and let $X_{0}^{\prime}$ be the space of bounded sequences summed to zero by $B$. That $c_{0}$ is a proper subspace of $X_{0}^{\prime}$ follows from the hypothesis that $c$ is a proper subspace of $X$ when $\lambda(B) \neq 0$, and follows from $[1$, p. 97] when $\lambda(B)=0$. By the theorem, and since $X_{0}^{\prime} \subseteq X$, there exists no bounded projection of $X$ onto $c_{0}$, but by $[5, \mathrm{p} .938]$, there exist bounded projections of $c$ onto $c_{0}$. The corollary is thus proved.

\section{REFERENCES}

1. R. P. Agnew, Convergence fields of methods of summability, Ann. of Math. (2) 46 (1945), 93-101.

2. S. Mazur and W. Orlicz, On linear methods of summability, Studia Math. 14 (1954), 129-160.

3. R. D. McWilliams, On projections of separable subspaces of $(m)$ onto $(c)$, Proc. Amer. Math. Soc. 10 (1959), 872-876.

4. R. S. Phillips, On linear transformations, Trans. Amer. Math. Soc. 48 (1940), 516-541.

5. A. Sobczyk, Projection of the space ( $m)$ on its subspace $\left(c_{0}\right)$, Bull. Amer. Math. Soc. 47 (1941), 938-947.

6. A. Wilansky, Topological divisors of zero and Tauberian theorems, Trans. Amer. Math. Soc. 113 (1964), 240-251.

Exeter University, Exeter, England 